\title{
Construire collectivement le savoir pour éduquer au patrimoine : l'exemple d'un parcours pédagogique innovant en territoire rural
}

\author{
Collectively building knowledge to educate about heritage: the \\ example of an innovative educational course in rural areas
}

\author{
Barbara Szafrajzen ${ }^{1}$ \\ ${ }^{1}$ Université d'Aix-Marseille, Institut Méditerrannéen en Sciences de l'Information et de la Communication \\ Barbara.szafrajzen@univ-amu.fr
}

RÉSUMÉ. Dans le contexte économique européen actuel, l'offre touristique française se doit de développer des services numériques innovants (Boiron, 2014). Cet enjeu est d'autant plus présent qu'il s'agit de s'inscrire dans une " culture visuelle numérique " (Catoir-Brisson, Jankeviciute, 2014), de continuer à préserver et à valoriser des gestes de patrimonialisation (Davallon, 2006), en repensant et en recontextualisant (Mucchielli, 2003) les conceptions traditionnelles de la construction des savoirs, sans volonté ancrée toutefois de mettre en place une évritable culture du numérique (Licoppe, 2009).La présente recherche souhaite comprendre comment un parcours pédagogique innovant (Corroy, Roche, Savignac, 2017) peut servir à valoriser un territoire rural et, dans le même temps, permettre à des élèves de s'éduquer au patrimoine en construisant leur savoir en situation culturellement marquée (Bouzon, 2010).

ABSTRACT. In the current European economic context, the French tourist offer must develop innovative digital services (Boiron, 2014). This stake is all the morepresent since it is a question of being part of a "digital visual culture" (Catoir-Brisson, Jankeviciute, 2014). This is still preserving an enhancing these gestures of heritage development (Davallon, 2006), by rethinking and recontextualizing (Mucchielli, 2003) the traditional conceptions of the construction of knowledge. This without however firmly established desire to set a true digital culture (Licoppe, 2009). This research aims to understand how an innovative educational path (Corroy, Roche, Savignac, 2017) can serve to enhance a rural territory and, at the same time, allow students to learn about heritage by building their knowledge in such a culturallybased situation (Bouzon, 2010).

MOTS-CLÉS. Sens, parcours pédagogique, innovation, patrimoine, construction collective du sens.

KEYWORDS. Meaning, educational path, innovation, heritage, collective construction of meaning ethics.

\section{Introduction}

Depuis plusieurs années, l'innovation numérique (Boiron, 2014) permet à la filière touristique de conserver son caractère attractif. Il s'agit de faciliter l'accès au patrimoine (Benhamou, Thesmar, 2011) et à la création contemporaine, dans une dynamique de démocratisation culturelle, de transmission des savoirs et d'éducation au patrimoine au service de la diversité de l'offre culturelle.

De nouveaux services émergent, conduisant à repenser les conceptions traditionnelles de la transmission en construction des savoirs, notamment au sein de parcours pédagogiques innovants en territoire rural. En sus de dynamiser l'offre touristique, ils offrent une qualification culturelle et patrimoniale d'excellence et sont un appui à l'économie locale : "A cet effet, les musées sont des institutions culturelles sélectionnant, conservant, possédant, documentant et exposant des patrimoines. » (Meunier, Soulier, 2010, p.22). Nous avons choisi de nous intéresser à la structure Argileum, La maison de la poterie située à Saint-Guilhem-Le-Désert Vallée de l'Hérault. Nous nous sommes demandé comment éduquer au patrimoine à travers un parcours pédagogique innovant en territoire rural. Pour répondre à cette problématique, la première partie de l'article présente les ancrages épistémologique et méthodologique de la recherche. La seconde partie expose ensuite le terrain de recherche avant d'analyser les apports des regards croisés à la frontière entre deux 
courants, les Sciences de l'éducation et de la formation et les Sciences de l'information et de la communication.

\section{Ancrages épistémologiques et méthodologiques}

La présente recherche est largement ancrée dans une position épistémologique dite de «convention constructiviste» (Le Moigne, 2003). En effet, sur la thématique des pratiques pédagogiques, ce positionnement se distingue des approches de type positiviste tels le courant behavioriste de la fin du XIXe siècle, qui ne traite «que les manifestations comportementales des processus psychologiques, en termes de stimuli et de réponses à l'environnement» (Danvers, 2009, p.67), ou encore le néobehaviorisme qui le prolonge par "l'introduction de certaines variables intermédiaires entre $S$ et $R$, comme la carte cognitive, la motivation » (Ibid). Le positionnement interprétativiste, quant à lui, est fondé sur une mise en contextualisation de la connaissance, sur des hypothèses phénoménologiques, sur une interdépendance entre le sujet et l'objet, sur une vision du monde social intentionnel et sur une orientation interactionniste qui rejette toute forme de déterminisme et «consiste avant tout à “ donner à voir "la réalité des acteurs étudiés » (GirodSeville, Perret, 2007, p.24). C'est en partie sur ce dernier aspect qu'une distinction s'opère, la genèse de la connaissance n'étant pas l'empathie mais la construction, avec pour corollaire le besoin de transformer cette connaissance. Le constructivisme est ainsi fondé sur l'idée que toute connaissance est construite par un apprenant sur la base d'une activité mentale ; c'est en réfléchissant et en se questionnant sur le monde que l'on construit cette vision du monde. Ces constructions peuvent parfois être très éloignées du monde existant (nous pensons notamment aux théories naïves des enfants) ; néanmoins, celles-ci s'étoffentet s'affinent de façon à devenir de plus en plus réalistes.

Ce courant de pensée est issu des travaux d'un certain nombre d'auteurs, dont Jean Piaget (18961980); à travers ses recherches, il montre comment un sujet construit sa vision du monde en donnant les détails de la genèse progressive des structures transcendantales. Selon lui, connaître consiste à agir sur le réel de façon à le transformer. Ainsi, l'individu devient acteur, voire « auteur » de sa connaissance « (...) dans la mesure où (...) l'individu, au moyen d'actions physiques et mentales par lesquelles il assimile le réel et s'accommode à lui, construit (et reconstruit) les concepts et les structures de son intelligence. ». (Vidal, 2004, p.22).

Le terme de «convention » est employé par Jean-Louis Le Moigne et «nous permet de disposer d'une représentation "généralement" acceptée de la convention épistémologique"classique"ou “institutionnelle" »(Le Moigne, 2003, p.45). Les fondements des épistémologies constructivistes sont au nombre de cinq. Selon Jean-Louis Le Moigne, ils constituent le fil conducteur reliant toutes formes de posture de convention constructiviste: le principe de représentativité, le principe d el'univers construit, le principe de projectivité ou d'interaction sujet-objet, le principe de l'argumentation générale et le principe d'action intelligente (Le Moigne, 1990). À travers ces fondements, il est entendu que la pratique pédagogique ne s'essaye pas, elle se vit et se consturit progressivement par l'acteur, en « situation en train de se faire » de construction du savoir, et plus précisément ici du patrimoine: «Les patrimoines sont des constructions culturelles issues d'une collectivité permettant d'asseoir, de revendiquer une identité culturelle et de témoigner de diverses formes d'authenticité. La collectivité assure aussi sa transmission aux générations futures. » (Davallon, 2002, p.62).

La notion de patrimoine est ici pensée et rattachée à celle de territoire : «Dans les villages, la culture et le patrimoine constituent des atouts à la fois formidables, limités et réalistes qu'il faut valoriser tout en veillant à conserver l'ancrage local, le risque systémique étant d'en externaliser les tenants et les aboutissants au point d'en déterriorialiser les spécificités. » (Lacour, 2014, p.792). De surcroît, cette notion de mémoire collective était déjà usitée il y a plusieurs années par des auteurs issus des sciences de l'information et de la communication. Mais, la définition de la 
notion de patrimoine peut aussi faire le lien avec des valeurs économiques. À ce titre, le rapport de Françoise Benhamou et David Thesmar se consacre aux politiques publiques de valorisation du patrimoine culturel de la France. Ces auteurs soulignent que «Le patrimoine est un outil de croissance et une résultante de la croissance ; il implique des coûts importants mais constitue un formidable levier pour le redressement d'une image et l'attractivité d'un lieu ou d'une région (...). Le patrimoine est d'abord un bien politique, c'est-à-dire un bien qui ne prend son sens que par rapport à des objectifs politiques : nationaliste (patrimoine national), idéologique (en lien avec des actes considérés comme fondateurs), éducatif (création d'un homme nouveau), ou identitaire (favoriser 1'adhésion de telle ou telle partie de la population)» (Benhamou \& Thesmar, 2011).

Fort de ces ancrages épistémologiques et théoriques, l'approche méthodologique choisie pour étudier ce terrain est celle d'une recherche qualitative en Sciences Humaines Sociales. Cette démarche méthodologique, rattachée au positionnement compréhensif, est habituellement appelée « démarche empirico-inductive » (Thiétart, 2007). Elle permet de construire progressivement nos réflexion et problématique, sans partir d'hypothèses a priori que nous aurions à valider sur le terrain: "En effet, la démarche est dite inductive, c'est-à-dire qu'elle cherche à explorer le réel, sans hypothèses de départ fortes, avec seulement un thème d'enquête, mais sans présupposés sur les résultats. Cela demande de la souplesse dans le déroulement de l'enquête, de technique de recueil de l'information, de populations à rencontrer ou de questionnements. » (Alami, Desjeux, GarabuauMoussaoui, 2009, p.25). De plus, le recours à plusieurs techniques de recueil de données minimise les effets pervers de chacune d'entre elles : "La triangulation peut être conçue comme une modalité particulière de plusieurs méthodes où l'objectif recherché est d'accrôttre la vraisemblance des conclusions d'une étude par l'obtention des résultats convergents obtenus par des méthodes différentes. » (Péladeau, Mercier, 1993, p.191). Ainsi, nous nous sommes immergés sur notre terrain de recherche durant un mois auprès des touristes qu'il est courant d'appeler dans la filière touristique des « séniors confirmés », constituant la principale clientèle en fin de saison. Nous avons tout d'abord réalisé des observations participantes auprès de ces visiteurs en situation de découverte du musée. Positionnée dans chacune des salles et nous nous présentions à eux lors lors de leur arrivée en leur demandant de «nous oubler et naviguer à leur convenance, faisant abstraction de notre présence »). À l'issue de leur visite, nous leur avons administré des questionnaires afin de recueillir leurs impressioons sur l'appropriation de ce média. «Par appropriation, nous entendons l'intégration créatrice d'éléments significatifs de cette culture dans la vie quotidienne des usagers et des collectivités (Proulx, 1998). L'appropriation constructive de cette culture apparaitrait alors comme une clé vitale de l'insertion des individus et des collectivités dans la "société du savoir" ". (Proulx, 2002, p.193). Pour mieux comprendre cela, il nous faut préciser les quatre conditions de réalisation de l'appropriation d'une technique: la maîtrise technique et cognitive de l'artefact, l'intégration significative de l'objet technique dans la pratique quotidienne de l'usager, l'usage répété de cette technologie, et l'appropriation sociale. Les questions avaient pour enjeu d'identifier les motifs de leur venue, leur connaissance antérieure du domaine de la poterie, leur ressenti sur le dispositif, leur représentation de l'interaction entre les objets numériques et la thméatique du musée, et leurs attentes et (in)satisfactions. Notre corpus, issu du croisement des données entre observations participantes et entretiens semi-directifs actifs, nous permet d'analyser la plue-value des services numériques pour éduquer au patrimoine et comprendre cette construction du savoir en situation de découverte du média.

\section{Terrain de recherche : un parcours pédagogique innovant en territoire rural}

L'Office de Tourisme Intercommunal de Saint-Guilhem-le-Désert Vallée de l'Hérault a créé en juillet 2011 la structure Argileum, La maison de la poterie. Les acteurs de ce projet de développement touristique en territoire rural ont eu pour ambition initiale de l'inscrire dans une triple perspective : dynamiser l'offre touristique dans un premier temps - et tenter de transformer le tourisme de passage en tourisme de séjour, tout en développant l'activité touristique hors saison-, 
offrir une qualification culturelle d'excellence dans un second temps (la Vallée de l'Hérault est labellisée PER : Projet d'Excellence Rurale, certification attestant de la qualité des projets innovants dans les territoires ruraux) et, enfin, être un appui à l'économie locale (en mettant en valeur les productions du village, la poterie, la mosaïque -qui a fait la renommée du village-, mais également la viticulture, très présente dans les ressources locales).

Ces ambitions reposent sur des enjeux forts de la gestion du Grand Site Saint-Guilhem-le-DésertGorges de l'Hérault. Il s'agit de concilier la mise en valeur du patrimoine respectueuse de l'identité du territoire et de son histoire, ces gestes de patrimonialisation (Davallon, 2006), le développement économique local, et l'amélioration des conditions de vie des habitants, au sein d'un projet touristique et culturel se voulant structuré et qualitatif. Ici, le musée a vocation à éduquer au patrimoine du territoire rural, soit la poterie. De nombreux chercheurs en Sciences de l'éducation se sont emparés de cette notion d'éducation au patrimoine ces dernières années (Pagoni \& TutiauxGuillon, 2012 ; Sauvé \& Girault, 2008 ; Musset, 2012, Barthes \& Alpe, 2015 ; etc.).

Pour Barthes et Alpe, l'éducation au patrimoine aurait aujourd'hui trois formes: «dans la première forme, elle s'intéresse à l'éducation le plus souvent formelle, mais pas exclusivement et elle concerne les connaissances "sur » les contenus patrimoniaux. (...). La deuxième forme est plus une éducation "par » qu'une éducation "sur». Elle vise à susciter une communauté de valeurs et d'identification à des spécificités terrioriales par le biais d'une culture commune émergente partagée (...). La troisième forme est une éducation "pour» le patrimoine. Si les objectifs sont clairement, c'est-à-dire susciter une communauté de valeurs, la formulation "pour» indique clairement une posture " utilitariste » de l'éducation. » (Barthes \& Alpe, 2015). Ici, l'équipement choisi est susceptible de s'adresser au plus grand nombre (grand public, itinérants culturels, enseignants, scolaires, séniors, etc.) de manière ludique, en permettant de découvrir -voire redécouvrir- l'univers de la poterie.

Dans une ambiance valorisant l'authenticité du XIXe siècle, le musée comprend l'ancien atelier Albe Sabadel, entièrement préservé et restauré, inscrit à l'inventaire supplémentaire de monuments historiques: "Centre d'interprétation novateur, interactif et ludique, consacré à la céramique, traditionnelle et moderne, artisanale et artistique, d'hier et d'aujourd'hui. Chaque année, un artiste est convié à revisiter librement ses jardins. Ces expositions constituent une valorisation commune et mutuelle de la transmission des savoir-faire, de la préservation d'un patrimoine, et de la création artistique contemporaine. ». (Extrait de la plaquette de présentation du musée). Argileum est un parcours pédagogique interactif alliant des moyens scénographiques issus de technologies récentes : des personnages historiques (Élie Sabadel, Jacques Hugol, Johan Salié, etc.) sont mis en scène par des moyens technologiques variés en muséologie (théâtre optique par système Dreamoc, un affichage holographique 3D à 4 côtés permettant de combiner un produit physique de grande taille avec un contenu 3D holographique), des projections sur écrans LCD permettent au visiteur une approche novatrice de la construction du savoir, des manipulations sur écrans tactiles ou encore un livre virtuel offrent un parcours se voulant plus ludique, sensoriel et interactif.

Ce parti pris scénographique est lié au cadre architectural des lieux, permettant de suivre les étapes de fabrication de la terre cuite vernissée, de la préparation de l'argile à la cuisson des objets produits, destinés principalement à l'usage domestiue ou architectural (tuiles, tuyaux, épis de faîtages, etc.), selon les pratiques des XIXe et XXe siècles. Ces mises en scènes se retrouvent dans plusieurs des salles et immergent le visiteur dans cette notion de construction du savoir et dans la création in situ: "la mise en scène de lieux de mémoire de travail, revient à désigner comme patrimoine des objets aupravant ordinaires et le sens se construit dans le geste même de la mise en public qui invite à rechercher des éléments qui correspondent aux besoins actuels $\gg$ (Gellereau \& Casemajor Lousta, 2009). Les objets de la collection renseignent également sur ce patrimoine culturel régional inhérent au mode de vie et à une époque («orjols »-les cruches caractéristiques de 
la région montpelliéraine-, « conçues », « gargoulettes », etc.) dont le visiteur est amené à découvrir la signification et les usages au cours de sa visite.

Le musée fait découvrir la vie des potiers, le savoir-faire et l'histoire de la céramique traditionnelle, moderne, artisanale et artistique, à travers un parcours couplé à des démonstrations d'un savoir-faire ancestral, le plaçant dans un environnement multisensoriel où de nombreux sens sont sollicités : la vie, le toucher, l'odorat, et l'ouïe. En s'inscrivant dans le territoire de la Communauté d'Agglomération Hérault-Méditerranée, cet espace devient telle une vitrine affichant les ressources et les richesses de ce bassin de séjour.

\section{Lecture communicationnelle}

Ce parcours pédagogique se veut un objet innovant en soi, en alliant notamment des moyens scénographiques issus de technologies récentes, des projections sur écrans LCD, des manipulations sur écrans tactiles, etc. De surcroît, l'éducation au patrimoine se veut centrée sur la construction du sens et la signification du sens émergeant, dans la mesure où l'émergence de ces significations ne peut se faire qu' " en situation en train de se faire » et est liée à l'utilisation de l'interactivité comme moyen d'émergence (Leleu-Merviel, 2008). Cette méthode d'analyse des phénomènes communicationnels est appelée la méthode de la sémiotique situationnelle (Mucchielli, 2003) ; elle permet de replacer toute action dans son contexte et d'analyser cette action au regard des enjeux, des positionnements, des normes, ou encore des éléments sensoriels faisant sens pour les acteurs en présence. En observant comment les acteurs agissent en situation à travers la réalisation de communications processus, l'analyste comprend comment la situation prend un sens spécifique pour l'acteur. Ces communications processus interviennent sur différents contextes de la situation et font émerger la construction du sens de la situation pour l'acteur concerné (Szafrajzen, 2011). En effet, le visiteur va pouvoir partager son ressenti lors de la visite du parcours pédagogique, l'amenant également à une construction collective du sens : «Le savoir devient une pratique collective, qui se développe dans la participation à des activités au sein d'une communauté qui façonne aussi l'identité de ses membres. ». (Morillon, Bouzon \& Caria, 2008, p.167). A ce titre, les observations participantes et les entretiens semi-directifs actifs que nous avons réalisés durant le mois de septembre 2014 permettant de comprendre de quelle manière ce parcours pédagogique innovant en territoire rural éduque à la notion de patrimoine.

Le corpus soulève l'importance des différents sens convoqués durant tout le parcours du visiteur ; ce dernier est invité à devenir acteur de son apprentissage (Szafrajzen, 2010) en convoquant quatre de ses différents sens. La vue, dans un premier temps, est bien entendue sollicitée lorsque le visiteur découvre les décors et les espaces, lorsqu'il lit les textes explicatifs ayant plusieurs supports et niveaux de lecture, susceptibles d'apporter des informations tant au novice qu'à l'amateur éclairé, à l'historien qu'au touriste, à l'archéologue qu'au collectionneur. L'ouïe, par ailleurs, est sollicitée dans chacune des salles à travers l'écoute des films et documentaires notamment. Le toucher est également très présent; en effet, après quelques secondes d'hésitations, nous observons que le visiteur ressent systématiquement le besoin de toucher les objets qui, pour la plupart, sont à sa portée. L'odorat enfin, est également partie prenante de la visite puisqu'une odeur de terre est présente dans chacune des salles et invite le visiteur à sentir les objets qu'il découvre. Sur le sens de l'odorat tout particulièrement, plusieurs visiteurs ont évoqué « une expérience unique », convoquant parfois même le terme « authentique », au sens de la préservation du monde marchand (Apostolakis, 2003).

Dans cet univers, l'éclairage joue également un rôle bien particulier ; il s'agit d'un parti pris de départ présidant à l'ensemble du traitement scénographique des espaces. Ces derniers souhaitent rester le plus possible dans des conditions de luminosité proches de celles de l'époque de fonctionnement, afin d'en préserver l'ambiance faisant partie de l'expérience vécue par le visiteur. Le respect des ouvertures historiques fait d'ailleurs partie des prescriptions émises par l'ABF 
(Architectes des Bâtiments de France) lors du calibrage du projet sur le bâtiment. Un atelier « dans son jus » fait partie de la collection; il est à la fois objet d'exposition et lieu d'accueil du parcours, et la scénographie en émane plutôt qu'elle ne lui est imposée. Ainsi, si les SIC permettent d'appréhender la complexité de cette situation (D'Almeida, 2001) afin de comprendre l'émergence du sens en situation (Szafrajzen, 2011). Il s'agit de comprendre comment un acteur, ici le visiteur du musée, en observant un phénomène, fait émerger des "communications généralisées significatives » (Mucchielli, 2003) et donne une signification aux objets découverts en les mettant en relation avec d'autres éléments de la situation (la problématique est celle de la genèse du sens partagé).

Nous comprenons alors que les règles, pour le visiteur, se construisent d'elles-mêmes, laissant place à une certaine créativité : «ce n'est pas parce que l'on se donne des règles qu'on apprend (...), mais parce que l'on apprend que l'onse donne des règles.» (Oustinoff, 2013, p.21). En affirmant cela, nous faisons référence à l'un des principes du constructivisme dit « faible » par Alex Mucchielli, celui de la «connaissance construite ». Dans cette idée, «le chemin de la connaissance n'existe pas a priori, il se construit en marchant» (Girod-Seville \& Perret, 2007, p.24), rejoingnant les fondements développés par Jean-Louis Le Moigne qui cite Giambattista Vico (1987) : «la vérité humaine est ce que l'homme connaît en le construisant, en le formant par ses actions » (Le Moigne, 1990, p.106). De ce fait, la construction des connaissances n'est pas linéaire dans la mesure où le public les construit au travers de chaque salle ponctuée de découvertes multiples et variées, convoquant sans cesse des sens différents : « La communication apparaît ainsi comme un processus complexe dans lequel la signification des messages n'est pas une donnée antérieure à l'interaction mais une construction en situation culturellement marquée qui dépend du déroulement du processus lui-même, dans le temps et dans l'espace. » (Bouzon, 2010, p.14).

Partant du constat que : "Personne n'éduque autrui, personne ne s'éduque seul, les hommes s'éduquent ensemble par l'intermédiaire du monde. » (Freire, 1967, p.62), nous choisissons une immersion sur le terrain pour comprendre cette construction du sens se réalisant de manière active et faisant émerger des significations propres à l'appropriation des connaissances du visiteur. La médiation numérique est donc mise au service de la valorisation d'un patrimoine, reconfigurant ainsi la conception traditionnelle des relations aux patrimoines. Ici, la culture du numérique est mise en exergue à travers l'expérience du visiteur confondue avec la découverte de l'histoire de la poterie et des objets présentés. À travers une approche qualitative (par triangulation méthodologique (Péladeau \& Mercier, 1993) fondée sur des observations participantes et des entretiens semidirectifs actifs, nous nous intéressons à l'interaction entre le visiteur et l'objet touristique (Cohen, 1988), à l'appropriation de cet objet, à la construction de son savoir : "En effet, d'une relation au savoir duale, on passe progressivement à une relation nettement plus complexe dans laquelle le savoir n'est plus seulement un obet préfabriqué hérité du passé et qu'il faut transmettre mais devient un enjeu culturel qu'il faut reconstruire sans cesse en fonction du passé bien sûr, mais surtout inscrit dans le présent et en prospectant sur l'avenir. »(Clénet \& Gérard, 1994, p.18).

Les différents éléments qui constituent le contexte de l'acteur découvrant le musée sont à la fois des entités humaines, idéelles et matérielles ; c'est ainsi que l'acteur est envisagé comme en interaction constante avec l'environnement dans lequel il évolue, soutenant l'idée de Latour : « (...) c'est parce qu'onles partage avec des non-humains que les interactions peuvent agir dans le temps et dans l'espace»(Latour, 2006, p.47). Nous pensons en effet que les acteurs construisent euxmêmes le sens donné à l'objet, et ce sens émerge à travers la connaissance qu'ils ont de cet objet, mais aussi la connaissance qu'ils s'en font, à travers leurs représentations, leurs idéaux, tout comme les relations avec les autres objets, les autres acteurs, et finalement leur environnement : « $(. .$. l'objet entre dans une série de mises en rapport et d'articulations avec d'autres objets qui sont déjà là, auxquels il emprunte des propriétés et ajoute les siennes. ». (Moscovici, 1976, p.61).

Nous envisageons ces différents points de vue comme émergence du sens et comme utiles à la compréhension de la situation d'apprentissage (nous insistons également ici sur la notion de 
« situation », dans le sens «d'acteurs en situation »). En effet, mettre en évidence les points de vue des acteurs, c'est aussi faire surgir les significations pour l'acteur, « significations » qui sont la clé de sa compréhension et donc de son apprentissage (Depover, Giordana \& Marton, 1998) : « (...) la mise en scène de lieux de mémoire de travail, revient à désigner comme patrimoine des objets auparavant ordinaires et le sens se construit dans le geste même de la mise en public qui invite à rechercher des éléments qui correspondent aux besoins actuels. » (Gellereau \& Casemajor Lousta, 2009, p.4).

\section{Conclusion}

À la frontière entre deux courants, les sciences de l'éducation et de la formation et les sciences de l'information et de la communication, cet article présente une pratique pédagogique innovante permettant de s'approprier un média traditionnel de la filière touristique, et ainsi d'éduquer au patrimoine. Pour ce faire, nous adoptons une démarche inductive et partons d'approches terrain en mettant en avant l'interdisciplinarité (Taddéi, 2013) -voire la transdisciplinarité-. Cette lecture communicationnelle d'un parcours pédagogique interactif alliant des moyens scénographiques issus de technologies récentes s'est centrée sur un corpus issu d'observations participantes et d'entretiens semi-directifs actifs auprès de visiteurs du musée de la poterie, Argileum.

Dans ce musée, l'éducation au patrimoine se veut centrée sur la construction du savoir « in situ » et la signification du sens émergeant, dans la mesure où l'émergence de ces significations ne peut se faire qu' « en situation de se faire ». En effet, le visiteur va pouvoir partager son ressenti lors de la visite du parcours pédagogique, l'amenant également à une construction collective du sens, au sens de la production et construction -ou sensemaking- (Weick, 1995). Les informations délivrées s'accumulent en strates au fil des espaces, selon une architecture interne propre à chaque visiteur, à partir d'un questionnement personnel suscité par les médias interactifs.

Cette lecture communicationnelle montre que la «novation est toujours plus efficace quand elle articule l'ancien et le nouveau, la mémoire et l'imagination.» (Lumbroso, 2017, p.99). En observant comment les acteurs agissent en situation, notre recherche comprend comment la situation prend un sens spécifique pour l'acteur. Ces communications processus interviennent sur différents contextes de la situation et font émerger la construction du sens de la situation pour l'acteur concerné. De fait, la construction des connaissances n'est pas linéaire et le public convoque sans cesse des sens différents (la vue, l'ouïe, le toucher, l'odorat) : « Ce processus de construction de sens est déclenché par l'inattendu, orienté vers l'action et sensible au contexte. » (Moinet, 2009, p.222). C'est enfin également une manière d'aborder l'espace territorial par les sens que les hommes ont voulu lui donner et permettre aux visiteurs d'y jouer un rôle.

Ce musée est donc une réponse apportée comme objet technologique mis au service de la valorisation et de l'éducation au patrimoine en territoire rural, devenant telle que vitrine affichant les ressources et les richesses de ce bassin de séjour, bassin touristique dans lequel le patrimoine de la poterie peut également servir de repère identifiable.

\section{Bibliographie}

Alami, S., Desjeux, D., Garabuau-Moussaoui, I. (2009). Les méthodes qualitatives. Paris: PUF.

Apostolakis, A. (2003). The convergence process in heritage tourism marketing, Annals of Tourism Research, 30/4, 795-812.

Barthes, A., Alpe, Y. (2015). L'éducation au patrimoine dans les aires territoriales protégées, une dimension de l'éducation au développement durable ? In DIEMER A. \& MARQUAT C., Éducation au développement durable. Enjeux et controverses, De Boeck. 
Benhamou, F., Thesmar, D. (2011). Valoriser le patrimoine culturel de la France. Rapport du Conseil d'Analyse Economique, La Documentation française.

Boiron, M. (2014), L'innovation en question(s). Tout a été dit, tout a été fait, il ne reste que l'avenir à inventer, Cavilam, 23-36.

Bouzon, A. (2010), Comunicación y organización. Orígenes y fundamentos de los trabajos científicos francófonos, in II Congreso Internacional de la Asociación Espaňola de Investigadores de la Comunicación (AE-IC), Comunicación y desarrollo en la era digital, 3 à 5 de febrero de 2010, Facultad de Ciencas de la Comunicación de la Universidad de Málaga, 22 p.

Catoir-Brisson, M.-J., Jankeviciute, L., (2014), «Entretien et méthodes visuelles: une démarche de recherche créative en sciences de l'information et de la communication », Sciences de la société [Online], 92/2014, 111-127, mis en ligne le 01 décembre 2014, consulté le 23 février 2018.

Clénet, J., Gérard, C. (1994). Partenariat et alternance en éducation : des pratiques à construire. Paris : L'Harmattan.

Corroy, L., Roche E., Savignac E. (2017). Education aux médias et pédagogies innovantes. Agence Universitaire de la Francophonie, Editions Publibook.

D’Almeida, N. (2001). Les promesses de la communication. Paris: PUF.

Danvers, F. (2009). S'orienter dans la vie : une valeur suprême? Essai d'anthropologie de la formation. Presses universitaires du Septentrion.

Davallon, J. (2006). Le Don du patrimoine : une approche communicationnelle de la patrimonialisation. Paris: Hermes Science-Lavoisier.

Davallon, J. (2002), Tradition, mémoire, patrimoine. Dans B. Schiele (dir.), Patrimoine et identités. Québec: Éditions Multimondes.

Depover, C., Giardina, M., Marton, P. (1998). Les environnements d'apprentissage multimédia. Paris: L'Harmattan.

Freire, P. (1967). Pédagogie des opprimés suivi de Conscientisation et révolution. Revue française de pédagogie, vol. $30, n^{\circ} 30,62-64$.

Gellereau, M., Casemajor Loustau, N. (2009), «Dispositifs de transmission et valorisation du patrimoine : l'exemple de la photographie comme médiation et objet de médiation», in Colloque international des sciences de l'information et de la communication, Interagir et transmettre, informer et communiquer : quelles valeurs, quelle valorisation?, Tunisie, http://archivesic.ccsd.cnrs.fr/sic_00426294, date de la dernière visite : 20 février 2015.

Girod-Seville, M, Perret, V. (2007), Fondements épistémologiques de la recherche. Dans R-A. Thietart, Méthodes de recherche en management. Paris : Dunod, $3^{\text {ème } e ́ d i t i o n . ~}$

Héas, S., Poutrain, V. (2003), "Les méthodes d'enquête qualitative sur Internet», Revue en ligne de sciences humaines et sociales, Ethnographiques.org, [Online], $\mathrm{n}^{\circ} 4$, novembre 2003, URL : http://www.ethnographiques.org/2003/Heas,Poutrain.html Consulté en mars 2015.

Huteau, M. (1996). Les problématiques de l'orientation. Actes du séminaire Pour le développement de la culture de l'orientation, Académie de Créteil, 5-6 octobre 1995, 13-22.

Lacour, C. et al. (2014). Introduction. Culture, patrimoine, savoirs : facteurs dynamiques de développement. Revue d'Economie Régionale \& Urbaine, 2014/5, 785-799.

Latour, B. (2006). Changer de société. Refaire de la sociologie. Paris: La Découverte.

Leleu-Merviel, S. (dir.). (2008). Objectiver l'humain? Volume 1, Qualification, quantification, Paris : Éditions Lavoisier/Hermès Sciences Publications, Coll. Ingénierie Représentationnelle \& Constructions de Sen.

Le Moigne, J-L. (1990). Épistémologie constructivistes et sciences de l'organisation. Dans A-C Martinet, Épistémologies et sciences de gestion, Paris: Economica.

Le Moigne, J-L. (2003). Le constructivisme. Modéliser pour comprendre. Tome 3, Paris: L’Harmattan.

Licoppe, C. (2009). L'évolution des cultures numériques. De la mutation du lien social à l'organisation du travail. Paris: Fyp éditions.

Lumbroso, O. (2017). Une pratique pédagogique en miroir pour éduquer à un média culturel numérique. Dans L. Corroy, E. Roche, E. Savignac, Éducations aux médias et pédagogies innovantes, Agence Universitaire de la Francophonie, Éditions Publibook, 87-99. 
Meunier, A., Soulier, V. (2010). Quel rapport à la culture et au patrimoine ? Un partenariat culturel en contexte muséal avec le milieu scolaire en francisation. Les Sciences de l'éducation - Pour l'Ere nouvelle, 4, vol.43, 19-48.

Moinet, N. (2009). De l'information utile à la connaissance stratégique : la dimension communicationnelle de l'intelligence économique. Communication \& organisation, [En ligne], URL: http://communicationorganisation.revues.org/855.

Morillon, L., Bouzon, A., Caria, M. (2008). Les acteurs de l'entreprise face à l'émergence des TIC : des pratiques plurielles. Dans J. Gerbault (dir), La langue du Cyberspace : de la diversité aux normes. Paris : l'Harmattan, 161172.

Moscovici, S. (1976). La psychanalyse : son image et son public. Paris : Presses Universitaires de France.

Mucchielli, A. (2003). La méthode du tableau panoramique pour l'étude des situations. CERIC : Montpellier 3, février 2003.

Oustinoff, M. (2013). (In)discipline et interdisciplinarité : des mots aux choses. Hermès, La Revue 3, n67, 21-24.

Peladeau, N., Mercier, C. (1993). Approches qualitative et quantitative en évaluation de programmes. Sociologie et sociétés [En ligne] URL : http://www.erudit.org/revue/socsoc/1993/v25/n2/001547ar.html.

Proulx, S. (2002). Trajectoires d'usages des technologies de communication : les formes d'appropriation d'une culture numérique comme enjeu d'une société du savoir. Annales des télécommunications, tome 57, n 3-4, Paris, 180-189.

Szafrajzen, B. (2010). Lecture communicationnelle de deux dispositifs d'apprentissage au sein d'un même département universitaire. Le cas de la licence en sciences de l'information et de la communication. Thèse de l’Université de Montpellier III: LERASS.

Szafrajzen, B. (2011). Réflexions autour de la méthode dite de la sémiotique situationnelle fondées sur une recherche en communication des organisations. Communication \& Organisation, n³9, 183-194.

Taddei, F. (2013). Pour un enseignement interdisciplinaire. La revue Interdisciplinarité : entre disciplines et indiscipline, ${ }^{\circ}$ 67, Hermès, CNRS éditions, 57-61.

Thietart, RA. (dir.) (2007). Méthodes de recherche en management. Paris : Dunod.

Vidal, F (2004). L'esprit piagétien : hommage international à Jean Piaget. Dans O. Houdé, C. Meljac (dir), Paris : Presses Universitaires de France, $2^{\mathrm{e}}$ éd. Rev.et augm.

Weick, E.K. (1995). Sensemaking in Organizations.Sage, Californie: Thousand Oaks.

Zakhartchouk, J.-M. (2017). Quelle pédagogie pour transmettre les valeurs de la République? Paris: ESF éditeur. 